\title{
The development of an evidence-based clinical checklist for the diagnosis of anterior knee pain
}

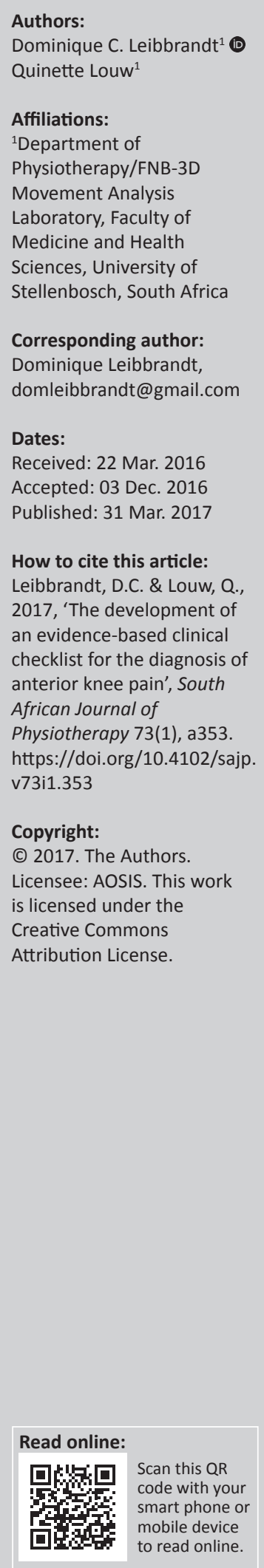

Background: Anterior knee pain (AKP) or patellofemoral pain syndrome is common and may limit an individual's ability to perform common activities of daily living such as stair climbing and prolonged sitting. The diagnosis is difficult as there are multiple definitions for this disorder and there are no accepted criteria for diagnosis. It is therefore most commonly a diagnosis that is made once other pathologies have been excluded.

Objectives: The aim of this study was to create an evidence-based checklist for researchers and clinicians to use for the diagnosis of AKP.

Methods: A systematic review was conducted in July 2016, and an evidence-based checklist was created based on the subjective and objective findings most commonly used to diagnose AKP. For the subjective factors, two or more of the systematic reviews needed to identify the factor as being important in the diagnosis of AKP.

Results: Two systematic reviews, consisting of nine different diagnostic studies, were identified by our search methods. Diagnosis of AKP is based on the area of pain, age, duration of symptoms, common aggravating factors, manual palpation and exclusion of other pathologies. Of the functional tests, squatting demonstrated the highest sensitivity. Other useful tests include pain during stair climbing and prolonged sitting. The cluster of two out of three positive tests for squatting, isometric quadriceps contraction and palpation of the patella borders and the patella tilt test were also recommended as useful tests to include in the clinical assessment.

Conclusion: A diagnostic checklist is useful as it provides a structured method for diagnosing AKP in a clinical setting. Research is needed to establish the causes of AKP as it is difficult to diagnose a condition with unknown aetiology.

\section{Introduction}

Knee pain affects about $70 \%$ of clients visiting the community health centres in the Western Cape (Parker \& Jelsma 2010). This alarming occurrence of knee problems is associated with moderate to high levels of disability.

Anterior knee pain (AKP) or patellofemoral pain syndrome (PFPS) frequently affects the knee joint and impairs functional ability (Parker \& Jelsma 2010).

The international incidence has been reported to be $25 \%-43 \%$ in sports injury clinics (Callaghan \& Selfe 2007; Witvrouw et al. 2000). AKP has a tendency to become chronic, and it has been estimated that $91 \%$ of patients diagnosed with AKP still experience symptoms four years after its onset. AKP is particularly common in adolescents, between the ages of 12 and 17 years (Rathleff et al. 2013), and may limit an individual's ability to perform common activities of daily living such as stair climbing and prolonged sitting (Nunes et al. 2013).

AKP is thought to be multifactorial in origin (Aminaka \& Gribble 2008). It also has the tendency to become chronic, especially in active individuals, adding an additional aspect of complexity to the treatment (Collins et al. 2012). There is agreement among recent reviews that conservative approaches are the preferred choice of treatment for AKP (Collins et al. 2012; McCarthy \& Strickland 2013). Surgical options such as distal realignment of the extensor mechanism, lateral retinacular release or debridement are generally only considered when conservative methods have failed or in the case of severe instability (McCarthy \& Strickland 2013).

The aetiology of AKP is not well understood. In addition, the aetiology may differ depending on whether symptoms are acute or chronic. There are a variety of pathways that could result in 
ongoing pain (psychological, pathophysiological, mechanical). However, the onset of the condition is hypothesised to involve excessive joint stress during activities that load the flexed knee joint. This patellofemoral joint stress is then transmitted through the cartilage, thereby exciting nociceptors in subchondral bone resulting in pain (Fulkerson 2002). Over time, this joint stress may result in articular cartilage pathology (Powers et al. 2014).

There are many definitions and synonyms for AKP. It is often used as an umbrella term for pathologies that cannot be classified as anything else, and therefore can include a variety of different pathologies. The term has been used interchangeably with PFPS, chondromalacia patellae, runner's knee, patellofemoral joint dysfunction and patella arthralgia (Collins et al. 2012; Cook et al. 2010; Lake \& Wofford 2011; Nunes et al. 2013). For the purpose of this article, we will be using the term 'anterior knee pain'.

Appendix 1 illustrates the range of definitions reported in systematic reviews.

The multiple definitions of AKP make accurate and standardised clinical diagnosis a challenging task for clinicians.

AKP is frequently defined as retropatellar or peripatellar pain, of more than three months duration, in the absence of intra-articular pathology, that is aggravated by activities that load a flexed knee joint (Crossley et al. 2001; Harvie, O'Leary \& Kumar 2011; Nunes et al. 2013; Prins \& van der Wurff 2009). The diagnosis of AKP is most commonly made based on the definition as well as the exclusion of other pathologies. However, this diagnostic procedure is vague and difficult to reproduce in a clinical setting.

The aim of this study was to create an evidence-based checklist for researchers and clinicians to use for the diagnosis of AKP.

\section{Methods}

\section{Study selection criteria}

English-only studies reporting on the clinical diagnostic tests for AKP were considered for inclusion. Due to the abundance of literature on $\mathrm{AKP}$, only systematic reviews were eligible for inclusion.

Studies describing the subjective information used for the diagnosis of AKP, such as the age of the patient, the duration of the symptoms, aggravating activities and previous history of trauma or other known knee injuries, were considered for inclusion.

Studies describing objective clinical tests used for the diagnosis of AKP were included. Radiographic procedures such as MRIs were excluded as these procedures cannot form part of a physiotherapy clinical assessment. For the same reason, arthroscopic procedures were also excluded.
The subjects of the studies included both genders. Exclusions were for studies that may have incorporated diagnoses of Osgood-Schlatter and osteoarthritis in participants younger than 18 years or older than 40 years. In addition, studies portraying knee abnormalities such as patella subluxation or intra-articular pathology were also omitted.

\section{Search strategy}

Publications from inception to July 2016, located in PubMed, Ebscohost (MEDLINE, CINAHL, SportDiscuss), Scopus and Science Direct, were accessed in library databases at the Medical Library at Stellenbosch University during July 2016.

The keywords used by the researcher (D.L.) in all the searches were: 'anterior knee pain', 'patellofemoral pain syndrome', 'diagnosis', 'clinical tests' and 'systematic reviews'. Searches were database-specific with $\mathrm{MeSH}$ terms for 'patellofemoral pain syndrome' used in search engines such as PubMed.

PRISMA Guidelines were followed with the reviewer (D.L.) screening the titles and abstracts of the first hits and consulting with the second reviewer (Q.L.) as needed. Both reviewers retrieved all potential complete texts independently and used the same criteria to decide which ones were relevant for inclusion in the review after having considered possible discrepancies in the texts. The individual diagnostic studies within the included reviews were then analysed.

\section{Methodological quality appraisal}

A clinical appraisal tool (CAT) for systematic reviews was used for the appraisal of included studies. This CAT comprises 10 questions assessing the methodological quality of the study and validity of the findings.

This CAT, as well as a detailed explanation of the criteria, can be found on the BMJ website (http://clinicalevidence.bmj. $\mathrm{com} / \mathrm{x} / \mathrm{set} / \mathrm{static} / \mathrm{ebm} /$ toolbox/665052.html) and is present in Appendix 2.

\section{Development of a diagnostic checklist}

An evidence-based checklist was created based on the subjective and objective findings. For the subjective factors, two or more of the systematic reviews were needed to identify the factor as being important in the diagnosis of AKP. For the objective factors, two or more of the reviews were needed to recommend the test based on either a sensitivity (more than $70 \%$ ) or a positive likelihood ratio (more than 5). A positive likelihood ratio of between 0 and 5 is considered to generate small but clinically important changes in probability (Nijs, Van Geel \& Van de Velde 2006). Clusters of tests found to improve diagnosis in any of the included reviews were also considered for the checklist.

\section{Results}

Two systematic reviews (Cook et al. 2012; Nunes et al. 2013), consisting of nine different diagnostic studies, were identified 
by our search methods. Of the nine diagnostic studies, four full texts were excluded as they used arthroscopic surgery for diagnosis and not clinical tests. A PRISMA flow chart is given in Figure 1.

The final checklist is presented in Appendix 3. Based on these studies, initial information that should be included in the subjective assessment includes age, area of pain, duration of symptoms, previous history of lower limb trauma or surgery and common aggravating factors. A flow chart of the diagnostic procedure is given in Figure 2.

As AKP is still largely a diagnosis of exclusion, patients should not be diagnosed with AKP if they are known to have any of the following pathologies: osteoarthritis, rheumatoid arthritis, patella fractures, patella subluxation and dislocation, fat pad impingement or bursitis, growth disorders such as Osgood-Schlatter, intra-articular pathology, patellar tendinitis, or referred pain from the lumbar spine or hip (Cook et al. 2010; Haim et al. 2006; Nijs et al. 2006; Sweitzer et al. 2010).

Objective tests can be divided into functional clinical tests, manual tests and exclusion of intra-articular pathologies.

Table 1 summarises the accuracy of commonly used diagnostic tests for AKP. Clinical functional tests that most commonly reproduce symptoms in patients with AKP are squatting, kneeling, stair climbing and prolonged sitting. Squatting is the most accurate functional test with a sensitivity of $91 \%$. Kneeling, stair ascent or descent and prolonged sitting follow with sensitivities of $84 \%, 75 \%$ and $72 \%$, respectively (Cook et al. 2010; Haim et al. 2006; Näslund et al. 2006; Nijs et al. 2006; Sweitzer et al. 2010).

It has been suggested that patients should present with pain in two or more of these activities in order to be diagnosed with AKP (Cook et al. 2012).

Of the manual tests considered, only the patella compression test (sensitivity of $83 \%$ ) and the patella tilt test (likelihood ratio $=5.4$ ) can be recommended as diagnostic tests for AKP (Haim et al. 2006; Näslund et al. 2006; Sweitzer et al. 2010).

On clinical appraisal of the two included systematic reviews (Cook et al. 2012; Nunes et al. 2013), both studies achieved scores of $8 / 10$, or $80 \%$. Therefore, these reviews can be considered to be of high methodological quality. Table 2 shows the scoring according to the CAT.

\section{Discussion}

In this article, we created a standardised method for the diagnosis of AKP based on a systematic review of the evidence.

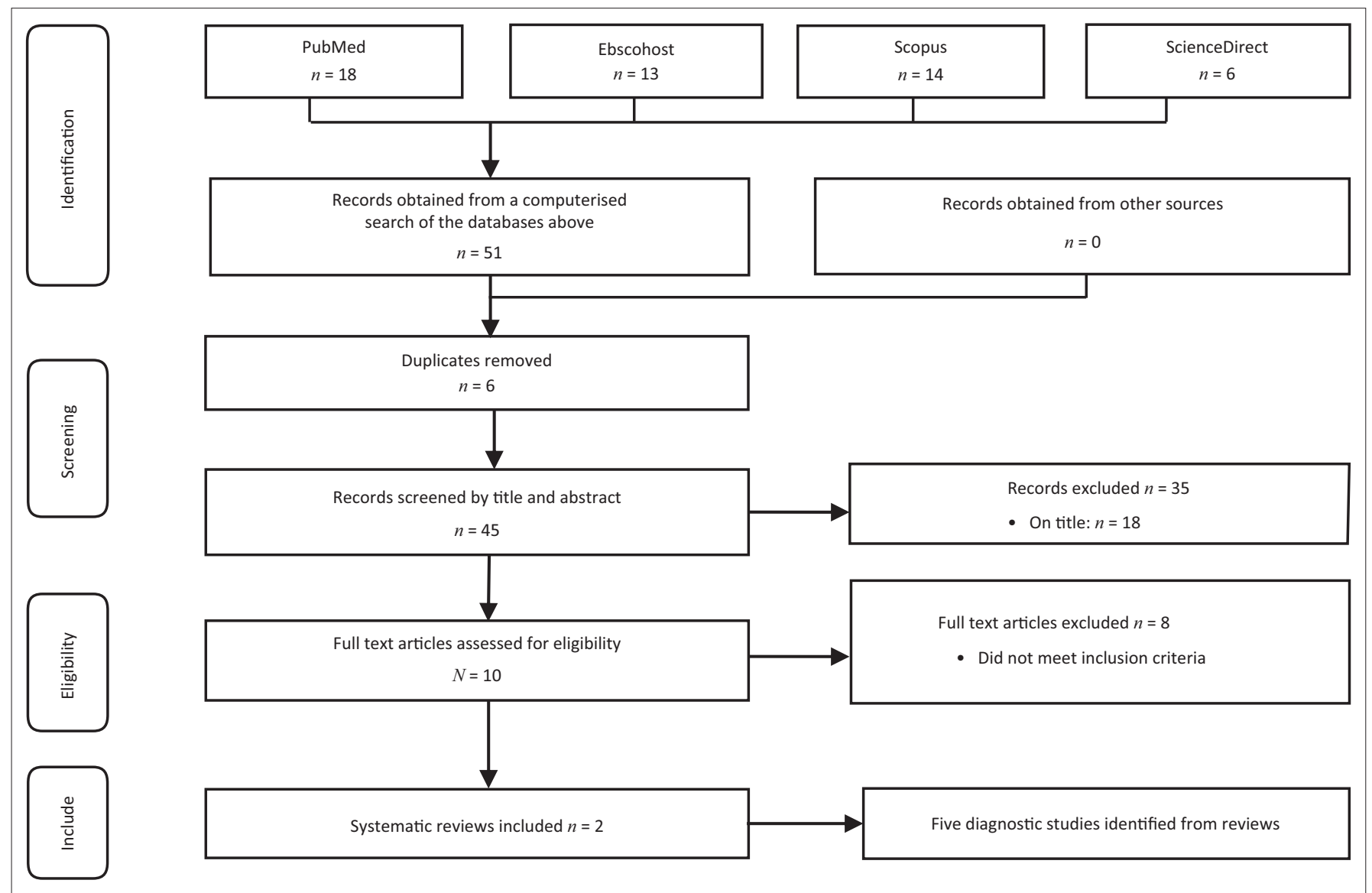

Source: www.prisma-statement.org/PRISMAStatement/FlowDiagram.aspx

$N$, total number.

FIGURE 1: PRISMA flow diagram of literature search. 


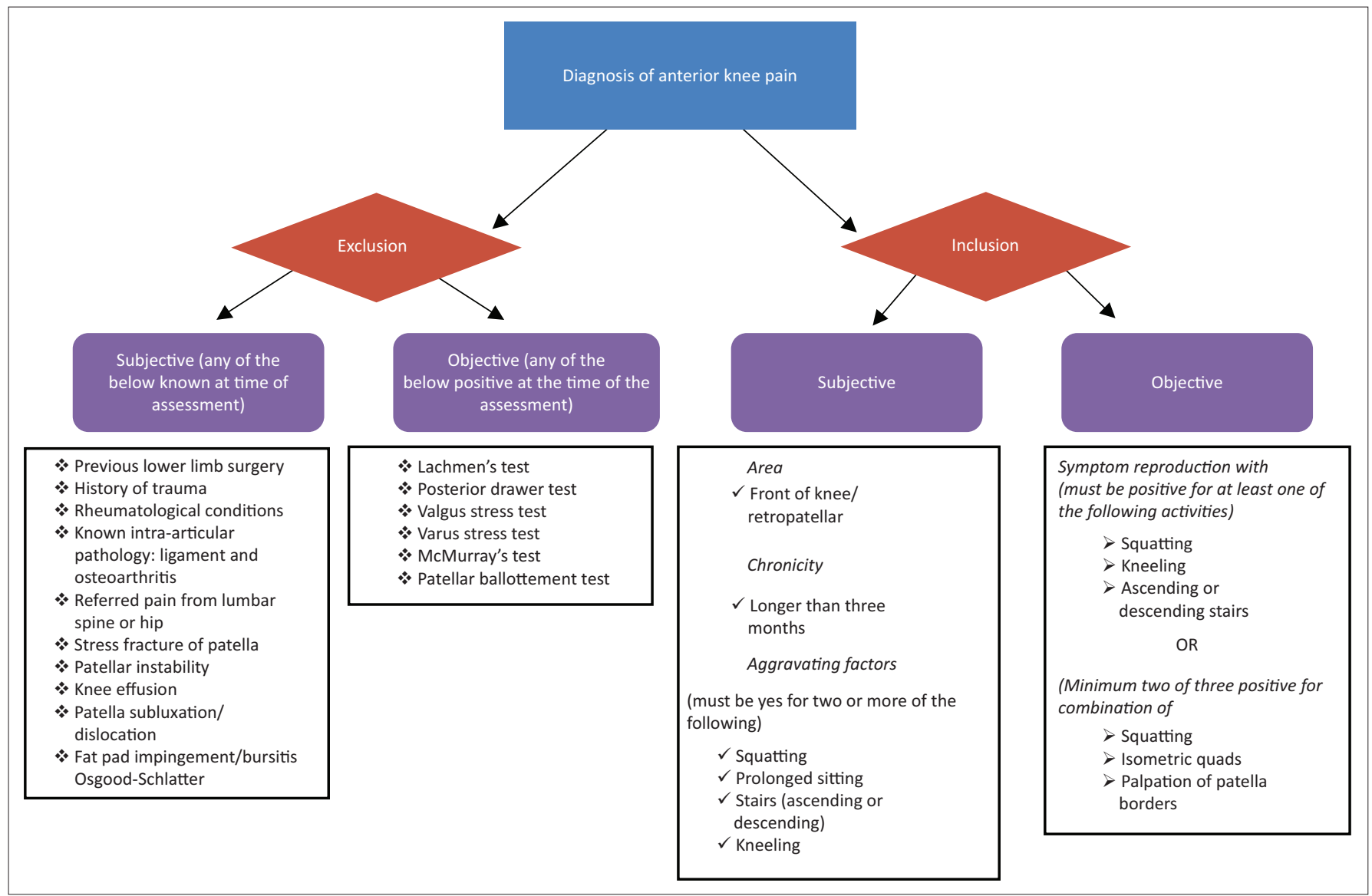

Source: Cook et al. 2012; Nunes et al. 2013

FIGURE 2: Flowchart demonstrating the process of diagnosis for anterior knee pain.

TABLE 1: Accuracy of diagnostic tests.

\begin{tabular}{lcccccc}
\hline Test & Sensitivity & Specificity & LR+ & LR- & PV+ & PV- \\
\hline Squatting & 91 & 50 & 1.8 & 0.2 & 79 & 74 \\
Kneeling & 84 & 50 & 1.7 & 0.3 & 79 & 61 \\
$\begin{array}{l}\text { Stairs - ascending } \\
\text { and descending }\end{array}$ & 75 & 43 & 1.3 & 0.6 & 73 & 46 \\
Prolonged sitting & 72 & 57 & 1.7 & 0.5 & 77 & 50 \\
Patella tilt test & 43 & 92 & 5.4 & 0.6 & 93 & 40 \\
$\begin{array}{l}\text { Patella } \\
\text { compression test }\end{array}$ & 83 & 18 & 1.0 & 1.0 & 63 & 38 \\
\hline
\end{tabular}

Source: Cook et al. 2010; Haim et al. 2006; Näslund et al. 2006; Nijs et al. 2006; Sweitzer et al. 2010

$\mathrm{LR}$, likelihood ratio; PV, predictive value.

TABLE 2: Quality of evidence.

\begin{tabular}{lcccccccc}
\hline Study & \multicolumn{3}{c}{ Cook et al. 2011 } & & \multicolumn{3}{c}{ Nunes et al. 2013 } \\
\cline { 1 - 2 } SR quality criteria & Yes & No & Can't tell & & Yes & No & Can't tell \\
\hline 1 & $\times$ & - & - & & $\times$ & - & - \\
2 & - & $\times$ & - & & - & $\times$ & - \\
3 & $\times$ & - & - & & $\times$ & - & - \\
4 & $\times$ & - & - & & $\times$ & - & - \\
5 & $\times$ & - & - & & $\times$ & - & - \\
6 & $\times$ & - & - & & $\times$ & - & - \\
7 & $\times$ & - & - & & $\times$ & - & - \\
8 & $\times$ & - & - & & $\times$ & - & - \\
9 & $\times$ & - & - & & $\times$ & - & - \\
10 & - & $\times$ & - & - & $\times$ & - \\
\hline Total & $8 / 10$ & - & - & $8 / 10$ & - & - \\
\hline
\end{tabular}

Source: http://clinicalevidence.bmj.com/x/set/static/ebm/toolbox/665052.html $\mathrm{SR}$, systematic review.
Diagnosis of AKP is based on the area of pain, age, duration of symptoms, common aggravating factors, manual palpation, and exclusion of other pathologies.

AKP can be defined as pain in the infrapatellar or retropatellar regions, in the absence of intra-articular pathology, that restricts activities of daily living that require knee flexion such as ascending or descending stairs, squatting and prolonged sitting (Cook et al. 2010; Haim et al. 2006; Näslund et al. 2006; Nijs et al. 2006; Sweitzer et al. 2010).

The subjective examination is important in the diagnosis of AKP. The interview should localise the pain, define the timing of onset and determine acute versus chronic versus overuse (Pećina \& Bojanić 1993). This information is important as it helps the clinician to rule out competing diagnoses. Extensor mechanism dysfunction is most commonly as a result of chronic repetitive trauma. AKP can also be patella subluxation or dislocation, ruptured patella or quad tendons. AKP during rest is often indicative of chondral lesions or dysfunctions (Post 1999; Smith et al. 2010).

A systematic review by Nunes et al. (2013) looked at five studies, that in total analysed 25 tests commonly used to diagnose AKP. The review concluded that there is no 
consistent evidence regarding the accuracy of commonly used diagnostic tests for AKP. However, the patellar tilt test (Haim et al. 2006) and the pain during squatting test (Cook et al. 2010) showed a strong tendency towards the PFPS diagnosis. The pain during squatting test demonstrated the highest sensitivity.

The other systematic review that was acquired through our search procedures (Cook et al. 2011) included nine studies; however, four were excluded as they made use of arthroscopy. The review included a variety of tests used to reproduce AKP including functional tests, patella mobility tests, special tests and the $Q$ angle test. Of these the functional tests, in particular squatting, stair climbing and prolonged sitting, demonstrated the highest accuracy.

Five diagnostic studies were identified from these two reviews (Cook et al. 2010; Haim et al. 2006; Näslund et al. 2006; Nijs et al. 2006; Sweitzer et al. 2010).

Nijs et al. (2006) investigated the validity of five clinical tests for AKP, including the vastus medialis coordination test, the patellar apprehension test, Waldron's test, Clarke's test and the eccentric step test.

In this study, the vastus medialis and patellar apprehension tests had a ratio of 2.26 and the eccentric step test scored 2.34. Waldron's test and Clarkes's test both scored below 2, thus questioning their validity. Limitations of the study included inability to standardise the amount of force used, the tests were performed in isolation and in reality these tests would be combined with other tests as part of a full subjective and objective clinical evaluation. The order of the tests also should have been standardised. Based on our criteria for inclusion, none of these tests is accurate enough to be considered for diagnosis.

Cook et al. (2010) explored the diagnostic accuracy of physical tests and functional activities commonly used to diagnose AKP. Clusters of functional findings and physical examination tests were also tabulated to determine combinations that improved diagnostic accuracy. Patients with intra-articular pathology were excluded. Measures used were manual compression of kneecap against femur (1) during rest and (2) during an isometric knee contraction, palpation of the posterio-medial and posterio-lateral borders of the patella, resisted isometric quadriceps femoris muscle contraction, squatting, stair climbing, kneeling and prolonged sitting. These measure were investigated as they are routinely used to measure AKP even though very few of these measures have been investigated for accuracy. The authors found that clusters may marginally improve accuracy. The cluster of two out of three positive tests for squatting, isometric quadriceps contraction and palpation of the patella borders scored the highest with a positive likelihood ratio of 4 . The authors recommended the use of this cluster of tests to diagnose AKP in a clinical assessment.
Individually, squatting, palpation, stepping down and the patella tilt test were recommended as useful tests to include in the clinical assessment.

Sweitzer et al. (2010) investigated the accuracy of patella mobility tests including superior-inferior patellar mobility, medial-lateral patellar mobility, patellar tendon mobility and patellar inferior pole tilt. However, all of these tests demonstrated poor sensitivity $(19 \%-63 \%)$ as well as positive likelihood ratios (1.4-1.9) and have therefore not been included in our checklist.

In a study by Näslund et al. in 2006, a physiotherapist and an orthopaedic surgeon examined 80 patients clinically diagnosed with AKP and referred for physiotherapy. The examination included a case history and a clinical examination. The four tests used in the clinical examination were the patella compression test, medial and lateral tenderness on extension, passive gliding of the patella and the $\mathrm{Q}$ angle test. The results indicated that the compression test demonstrated the highest sensitivity (83\%), but none of the tests could predict findings seen in radiographic examinations. The authors suggested that the $\mathrm{Q}$ angle test can no longer be considered a reliable test in diagnosing $\mathrm{AKP}$, as it shows great inter- and intra-observer variability. This is in agreement with a recent systematic review of prospective studies that demonstrated that the $\mathrm{Q}$ angle is not a risk factor for $\mathrm{AKP}$, thus questioning its relevance (Smith, Hunt \& Donell 2008). The authors (Näslund et al. 2006) suggested the AKP is still ultimately a diagnosis of exclusion as it is a term used for knee pain that can be attributed to multiple causes. Therefore, more research on pathophysiology needs to be done.

A very important aspect of diagnosis for AKP is the exclusion of intra-articular pathologies. These include ligaments such as ACL, PCL, MCL and LCL and the meniscii (medial and lateral). The most accurate tests to achieve this have been given in Table 3 (Benjaminse, Gokeler \& van der Schans 2006; Day, Fox \& Paul-Taylor 2009; Malanga et al. 2003; Nijs et al. 2006). Based on this, we have chosen to include the anterior drawer test, the posterior drawer test, the valgus stress test, the varus stress test, McMurray's test and the patellar ballottement test in our checklist for the purpose of exclusion.

TABLE 3: Most accurate tests for exclusion of intra-articular pathology.

\begin{tabular}{llcc}
\hline Test & Structure & Sensitivity (\%) & Specificity (\%) \\
\hline Lachmen's & $\mathrm{ACL}$ & 85 & 94 \\
Anterior drawer & $\mathrm{ACL}$ & 92 & 91 \\
Posterior drawer & $\mathrm{PCL}$ & $51-100$ & 99 \\
Valgus stress & $\mathrm{MCL}$ & $86-96$ & Not reported \\
Varus stress & $\mathrm{LCL}$ & 25 & Not reported \\
Pivot shift & Meniscus & 24 & 98 \\
McMurray's & Meniscus & $16-58$ & $77-98$ \\
Apley's grind & Meniscus & $13-16$ & $80-90$ \\
Patella ballottement & Effusion & 32 & 100 \\
\hline
\end{tabular}

Source: Benjaminse et al. 2006; Day et al. 2009; Malanga et al. 2003; Nijs et al. 2006 
The two reviews used for the creation of our evidence-based checklist were both of high quality. The reviews evaluated the quality of the included studies and took this into consideration when making the recommendations. Consequently, we can be confident that the checklist is based on high-quality evidence.

Nevertheless, in order to improve on this evidence, it is necessary to establish possible causes of AKP. Causes are believed to be multifactorial, and diagnosis is still largely a diagnosis of exclusion in a specific population of younger active people. Subgroups of individuals with AKP and aetiology may vary.

\section{Conclusion}

AKP can be defined as retro- or peri-patellar pain, of more than three months duration, in the absence of intra-articular pathology, that is aggravated by activities that load a flexed knee joint (Crossley et al. 2001; Harvie et al. 2011; Nunes et al. 2013; Prins \& van der Wurff 2009). The diagnosis of AKP is made based on the definition as well as the exclusion of other pathologies. There are many clinical tests used to diagnose $\mathrm{AKP}$; however, there is no standard method to diagnose AKP and many of the tests are not accurate. A diagnostic checklist is useful as it provides a structured method for diagnosing AKP in a clinical setting. Research is needed to establish the causes of AKP as it is difficult to diagnose a condition with unknown aetiology.

\section{Acknowledgements}

The authors wish to acknowledge that this work is based on the research supported in part by the National Research Foundation (NRF) of South Africa (Grant number CSUR1309 0332637). They would also like to express gratitude towards Stellenbosch University for the use of their facilities. The study would not have been possible without the input of these institutions.

\section{Competing interests}

The authors declare that they have no financial or personal relationships that may have inappropriately influenced them in writing this article.

\section{Authors' contributions}

D.C.L. and Q.L. screened the articles for inclusion, planned the article and critically appraised the included reviews. D.C.L. performed the searches, extracted data, compiled the checklist and drafted the original article. Q.L. edited the article and approved the final draft.

\section{References}

Aminaka, N. \& Gribble, P., 2005, 'A systematic review of the effects of therapeutic taping on patellofemoral pain syndrome,' Journal of Athletic Training 40(4), 341-351.

Aminaka, N. \& Gribble, P.A., 2008, 'Patellar taping, patellofemoral pain syndrome, lower extremity kinematics, and dynamic postural control', Journal of Athletic Training 43(1), 21-28.
Barton, C.J., Webster, K.E. \& Menz, H.B., 2008, 'Evaluation of the scope and quality of systematic reviews on nonpharmacological conservative treatment for patellofemoral pain syndrome,' Journal of Orthopaedic \& Sports Physical Therapy 38(9), 529-541. https://doi.org/10.2519/jospt.2008.2861

Benjaminse, A., Gokeler, A. \& Van der Schans, C.P., 2006, 'Clinical diagnosis of an anterior cruciate ligament rupture: A meta-analysis', Journal of Orthopaedic \& Sports Physical Therapy 36(5), 267-288. https://doi.org/10.2519/jospt.2006. 2011

Callaghan, M.J. \& Selfe, J., 2007, 'Has the incidence or prevalence of patellofemoral pain in the general population in the United Kingdom been properly evaluated?' Physical Therapy in Sport 8(1), 37-43. https://doi.org/10.1002/14651858. cd006717.pub2

Callaghan, M.J. \& Selfe, J., 2012, 'Patellar taping for patellofemoral pain syndrome in adults,' The Cochrane Library 4, CD006717.

Collins, N.J., Bierma-Zeinstra, S.M., Crossley, K.M., Van Linschoten, R.L., Vicenzino, B. \& Van Middelkoop, M., 2012, 'Prognostic factors for patellofemoral pain: A multicentre observational analysis', British Journal of Sportsmedicine 47 , 227-233.

Cook, C., Hegedus, E., Hawkins, R., Scovell, F. \& Wyland, D., 2010, 'Diagnostic accuracy and association to disability of clinical test findings associated with patellofemoral pain syndrome', Physiotherapy Canada 62(1), 17-24. https://doi.org/10.3138/ physio.62.1.17

Cook, C., Mabry, L., Reiman, M.P. \& Hegedus, E.J., 2012, 'Best tests/clinical findings for screening and diagnosis of patellofemoral pain syndrome: A systematic review, Physiotherapy 98(2):93-100.

Crossley, K., Bennell, K., Green, S. \& McConnell, J., 2001, 'A systematic review of physical interventions for patellofemoral pain syndrome,' Clinical Journal of Sport Medicine 11(2), 103-110.

Day, R.J., Fox, J.E. \& Paul-Taylor, G., 2009, Neuromusculoskeletal clinical tests: A clinician's guide, Elsevier Health Sciences. Cardiff University, Cardiff.

Fulkerson, J.P., 2002, 'Diagnosis and treatment of patients with patellofemoral pain', The American Journal of Sportsmedicine 30(3), 447-456.

Haim, A., Yaniv, M., Dekel, S. \& Amir, H., 2006, 'Patellofemoral pain syndrome: Validity of clinical and radiological features', Clinical Orthopaedics and Related Research $451,223-228$

Harvie, D., O'Leary, T. \& Kumar, S., 2011, 'A systematic review of randomized controlled trials on exercise parameters in the treatment of patellofemoral pain: What works', Journal of Multidisciplinary Healthcare 4, 383-392.

Heintjes, E., Berger, M.Y., Bierma-Zeinstra, S.M., Bernsen, R.M., Verhaar, J.A. \& Koes, B.W., 2003, 'Exercise therapy for patellofemoral pain syndrome,' Cochrane Database System Review 4, CD003472. https://doi.org/10.1016/s0031-9406 (05)60488-9

Heintjes, E., Berger, M.Y., Bierma-Zeinstra, S.M., Bernsen, R.M., Verhaar, J.A. \& Koes, B.W., 2004, 'Pharmacotherapy for patellofemoral pain syndrome.' Cochrane Database System Review 3, CD003470. https://doi.org/10.1002/14651858. cd003470.pub2

Lake, D.A. \& Wofford, N.H., 2011, 'Effect of therapeutic modalities on patients with patellofemoral pain syndrome: A systematic review', Sports Health: A Multidisciplinary Approach 3(2), 182-189. https://doi.org/10.1177/19417 38111398583

Lankhorst, N.E., Bierma-Zeinstra, S.M. \& Van Middelkoop, M., 2012, 'Risk factors for patellofemoral pain syndrome: A systematic review,' Journal of Orthopaedic \& Sports Physical Therapy 42(2), 81-112. https://doi.org/10.2519/jospt.2012.3803

Malanga, G.A., Andrus, S., Nadler, S.F. \& McLean, J., 2003, 'Physical examination of the knee: A review of the original test description and scientific validity of common orthopedic tests', Archives of Physical Medicine and Rehabilitation 84(4), 592-603.

McCarthy, M.M. \& Strickland, S.M., 2013, 'Patellofemoral pain: An update on diagnostic and treatment options', Current Reviews in Musculoskeletal Medicine 6(2), 188-194

Näslund, J., Näslund, U.B., Odenbring, S. \& Lundeberg, T., 2006, 'Comparison of symptoms and clinical findings in subgroups of individuals with patellofemoral pain', Physiotherapy Theory and Practice 22(3), 105-118.

Nijs, J., Van Geel, C. \& Van de Velde, B., 2006, 'Diagnostic value of five clinical tests in patellofemoral pain syndrome', Manual Therapy 11(1), 69-77.

Nunes, G.S., Stapait, E.L., Kirsten, M.H., De Noronha, M. \& Santos, G.M., 2013, 'Clinical test for diagnosis of patellofemoral pain syndrome: Systematic review with metaanalysis', Physical Therapy in Sport 14(1), 54-59. https://doi.org/10.1016/j. ptsp.2012.11.003

Parker, R. \& Jelsma, J., 2010, 'The prevalence and functional impact of musculoskeletal conditions amongst clients of a primary health care facility in an under-resourced area of Cape Town', BMC Musculoskeletal Disorders 11(1), 1.

Pećina, M. \& Bojanić, I., 1993, Overuse injuries of the musculoskeletal system, CRC Press, Boca Raton, FL.

Post, W.R., 1999, 'Current concepts clinical evaluation of patients with patellofemoral disorders', Arthroscopy: The Journal of Arthroscopic \& Related Surgery 15(8), 841-851. https://doi.org/10.1053/ar.1999.v15.015084

Powers, C.M., Ho, K.Y., Chen, Y.J., Souza, R.B. \& Farrokhi, S., 2014, 'Patellofemoral joint stress during weight-bearing and non-weight-bearing quadriceps exercises', Journal of Orthopaedic \& Sports Physical Therapy 44(5), 320-327. https://doi. org/10.2519/jospt.2014.4936

Prins, M.R. \& Van Der Wurff, P., 2009, 'Females with patellofemoral pain syndrome have weak hip muscles: A systematic review', Australian Journal of Physiotherapy 55(1), 9-15. 
Rathleff, M.S., Roos, E.M., Olesen, J.L. \& Rasmussen, S., 2013, 'High prevalence of daily and multi-site pain - A cross-sectional population-based study among 3000 and multi-site pain - A cross-sectional pop
Danish adolescents,' BMC Pediatrics 13(1),191.

Smith, T.O., Davies, L., Chester, R., Clark, A. \& Donell, S.T., 2010, 'Clinical outcomes of rehabilitation for patients following lateral patellar dislocation: A systematic review', Physiotherapy 96(4), 269-281. https://doi.org/10.1016/j.physio.2010. 02.006

Smith, T.O., Hunt, N.J. \& Donell, S.T., 2008, 'The reliability and validity of the Q-angle: A systematic review', Knee Surgery, Sports Traumatology, Arthroscopy 16(12) 1068-1079. https://doi.org/10.1007/s00167-008-0643-6
Sweitzer, B.A., Cook, C., Steadman, J.R., Hawkins, R.J. \& Wyland, D.J., 2010, 'The interrater reliability and diagnostic accuracy of patellar mobility tests in patients with anterior knee pain', The Physician and Sportsmedicine 38(3), 90-96.

Waryasz, G.R. \& McDermott, A.Y., 2008, 'Patellofemoral pain syndrome (PFPS): A systematic review of anatomy and potential risk factors', Dynamic Medicine 7(1), 9.

Witvrouw, E., Lysens, R., Bellemans, J., Cambier, D. \& Vanderstraeten, G., 2000, 'Intrinsic risk factors for the development of anterior knee pain in an athletic population a two-year prospective study', The American Journal of Sports Medicine 28(4), 480-489. 


\section{Appendix 1}

TABLE 1-A1: Definitions and synonyms for AKP.

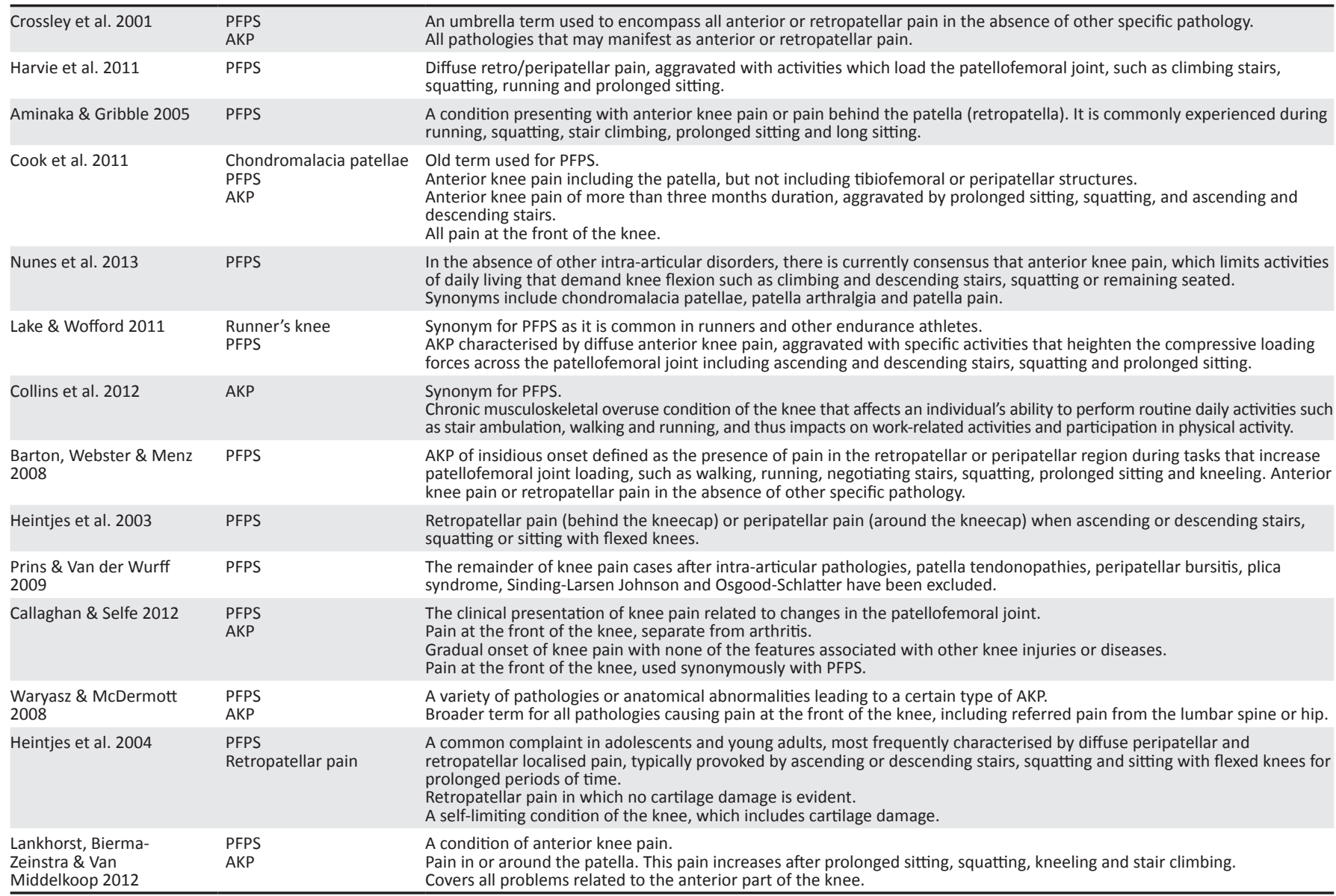

AKP, anterior knee pain; PFPS, patellofemoral pain syndrome. 


\section{Appendix 2}

TABLE 1-A2: Framework for assessing systematic reviews.

Does the SR explicitly report and perform a comprehensive and reproducible literature search?

Does the SR formulate a clearly focused question?

Does the SR's methods section explicitly state the basis for inclusion or exclusion of primary RCTs?

Does the SR report data from primary RCTs (e.g. size, interventions used, results from individual RCTs)

Does the SR assess the methodological quality of primary studies, and take these into account where necessary?

Meta-analysis: does the SR combine primary studies appropriately?

Meta-analysis: does the SR state how results are combined statistically?

Meta-analysis: does the SR report absolute numbers as well as appropriate summary statistics?

Does the SR discuss the reasons for any variations or heterogeneity between individual RCTs and overall results?

Does the SR report on the clinical relevance or importance of the results?

$\mathrm{RCT}$, randomised controlled trial; SR, systematic review. 


\section{Appendix 3}

\section{Checklist for diagnosis of anterior knee pain.}

\section{SUBJECTIVE INFORMATION:}

Age (must be yes)

$14-50^{1,2,3,4,5}$

Area (must be yes)

Front of knee or retropatella $a^{1,2,3,4,5}$

Chronicity

Longer than three months ${ }^{1,3,5}$

Aggravated by (must be yes for two or more of the following)

\begin{tabular}{|l|l|l|}
\hline Squatting ${ }^{1,2,3,4,5}$ & & \\
\hline Prolonged sitting ${ }^{1,2,3,4,5}$ & & \\
\hline Stairs (ascending or descending) (1,2,3,4,5 $^{1,3,5}$ & & \\
\hline Kneeling $^{1,2,3,4,5}$ & & \\
\hline
\end{tabular}

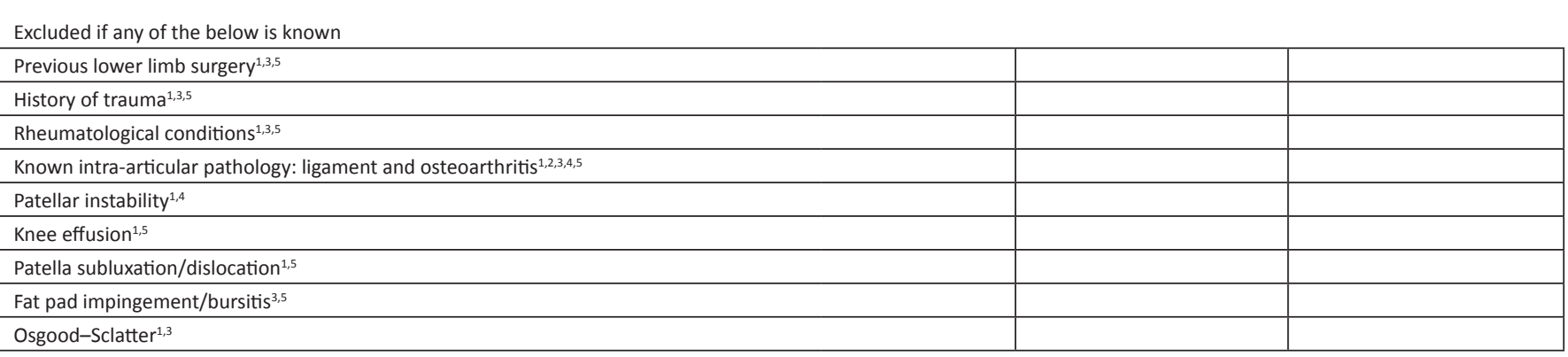

\section{OBJECTIVE TESTS:}

Symptom reproduction with (must be positive for at least one of the following activities)

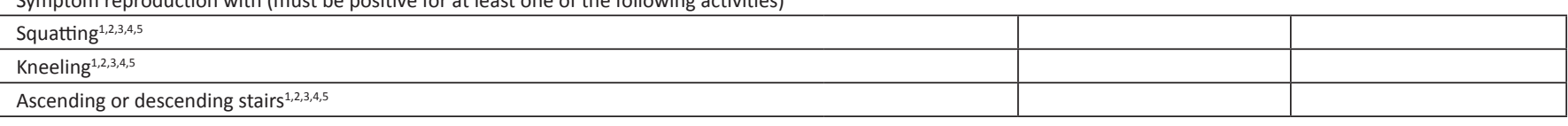

Positive for at least one of the following

Patella compression test ${ }^{1,4}$

Patella tilt test ${ }^{1,4}$

OR

(Minimum two out of three) positive for combination of

\section{Squatting ${ }^{3}$}

Isometric quads ${ }^{3}$

Palpation of patella borders ${ }^{3}$

\section{Excluded if positive for}

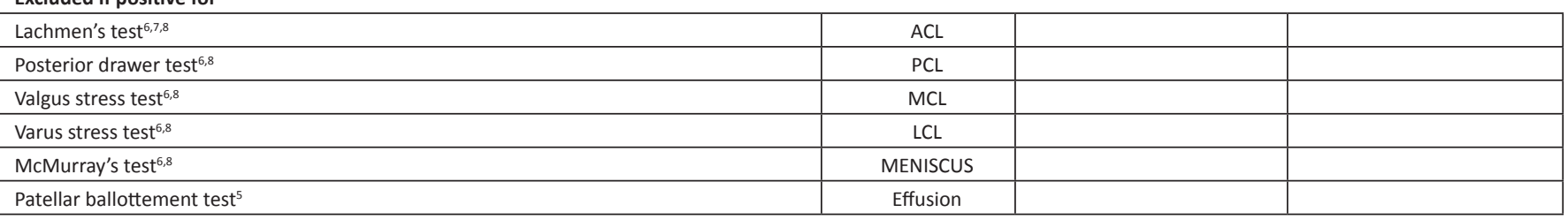

\section{References}

1. Haim, A., Yaniv, M., Dekel, S. \& Amir, H., 2006, 'Patellofemoral pain syndrome: Validity of clinical and radiological features', Clinical Orthopaedics and Related Research 451, 223-228.

2. Näslund, J., Näslund, U.B., Odenbring, S. \& Lundeberg, T., 2006, 'Comparison of symptoms and clinical findings in subgroups of individuals with patellofemoral pain', Physiotherapy Theory and Practice 22(3), 105-118.

3. Cook, C., Hegedus, E., Hawkins, R., Scovell, F. \& Wyland, D., 2010, 'Diagnostic accuracy and association to disability of clinical test findings associated with patellofemoral pain syndrome', Physiotherapy Canada 62(1),17-24.

4. Sweitzer, B.A., Cook, C., Steadman, J.R., Hawkins, R.J. \& Wyland, D.J., 2010 'The inter-rater reliability and diagnostic accuracy of patellar mobility tests in patients with anterior knee pain', The Physician and Sportsmedicine 38(3), 90-96.

5. Nijs, J., Van Geel, C. \& Van de Velde, B., 2006, 'Diagnostic value of five clinical tests in patellofemoral pain syndrome', Manual Therapy 11(1), 69-77.

6. Benjaminse, A., Gokeler, A. \& Van der Schans, C.P., 2006, 'Clinical diagnosis of an anterior cruciate ligament rupture: A meta-analysis', Journal of Orthopaedic \& Sports Physical Therapy 36(5), 267-288.

7. Day, R.J., Fox, J.E. \& Paul-Taylor, G., 2009, Neuromusculoskeletal clinical tests: A clinician's guide, Elsevier Health Sciences, Cardiff University, Cardiff.

8. Malanga, G.A., Andrus, S., Nadler, S.F. \& McLean, J., 2003, 'Physical examination of the knee: A review of the original test description and scientific validity of the knee: A review of the original test description and scientific validity of
common orthopedic tests', Archives of Physical Medicine and Rehabilitation 84(4), 592-603. 